\title{
INCIDENCIA Y ASOCIACIONES DE ARTERIA UMBILICAL ÚNICA EN RECIÉN NACIDOS. MATERNIDAD DEL HOSPITAL CLIINICO DE LA UNIVERSIDAD DE CHILE, 1998-2010
}

\author{
Julio Nazer H. ${ }^{1}$, Catalina Jarpa ${ }^{2}$, Lucía Cifuentes O. ${ }^{2}$ \\ ${ }^{1}$ Unidad de Neonatología, Maternidad Hospital Clínico de la Universidad de Chile. ${ }^{2}$ Instituto de Ciencias Biomédicas, \\ Facultad de Medicina Universidad de Chile.
}

a Alumna, Escuela de Medicina, Universidad de Chile.

\section{RESUMEN}

Antecedentes: La presencia de 2 vasos en el cordón umbilical es una anomalía con incidencia de $1 \%$ de todos los recién nacidos. Se asocia frecuentemente con restricción del crecimiento intrauterino (RCIU), malformaciones estructurales mayores y cromosómicas y prematuridad. Constituye un factor de riesgo que aumenta la mortalidad fetal tardía y neonatal. Objetivos: Establecer la prevalencia al nacimiento de arteria umbilical única (AUU), la magnitud de las asociaciones con malformaciones congénitas (MFC), RCIU, prematuridad y su influencia en la morbimortalidad neonatal. Método: Se usó la base de datos que el Estudio Colaborativo Latino Americano de Malformaciones Congénitas (ECLAMC) tiene en la maternidad del Hospital Clínico de la Universidad de Chile. Incluyó a todos los recién nacidos vivos (RN) y mortinatos con peso de nacimiento 500 gramos o más en el período mayo 1998 a junio 2010. Se comparó algunas variables demográficas entre los casos y sus controles. Resultados: En 22.011 nacimientos ocurridos hubo 65 RN con AUU (0,3\%), 9 eran mortinatos (13,8\%), 88,9\% de ellos presentaban RCIU. Eran prematuros $55,4 \%$ de los RN. El $60 \%$ de los casos de AUU estaban asociados a MFC mayores, destacando las del aparato genitourinario $(35,3 \%)$ y las cardiovasculares $(29,4 \%) ; 23,8 \%$ eran parte de un síndrome, donde dominaban la trisomía 13 y 18 (12 casos, 18,5\%). De los RN vivos fallecieron $13(23,2 \%), 8(61,5 \%)$ presentaban RCIU. Conclusión: La AUU es un factor de alto riesgo de asociación con RCIU, MFC mayores estructurales y/o cromosómicas, prematuridad y de un aumento de la morbimartalidad neonatal.

\section{PALABRAS CLAVE: Arteria umbilical única, malformaciones congénitas}

\section{SUMMARY}

Background: The presence of only two blood vessels in the cord blood is a congenital anomaly that has an incidence about $1 \%$ among all newborns. Frequently, it is associated with intrauterine growth restriction (IUGR), major congenital malformations (MCMF), chromosome anomalies and prematurity. It represents a risk factor that increases late foetal and neonatal mortality and morbidity. Aims: To estimate the prevalence at birth of unique umbilical artery (UUA), to know its association with congenital anomalies, IUGR, prematurity and to evaluate its influence in neonatal morbidity and mortality. Methods: We studied data registered in the Latin American Study of Congenital Malformations (ECLAMC) from the Maternity of the Clinical Hospital of University of Chile from May 1998 and June 2010. All newborns (alive and stillbirths) with a birth weigh 
$>500 \mathrm{~g}$ were included. Demographic variables were compared between cases and controls. Results: 65 newborns with UUA (0.3\%) was found among the 22.011 births registered: 9 was stillbirths $(13.8 \%), 88.9 \%$ of them had IUGR and $55.4 \%$ were premature. $60 \%$ of cases has another MCMF (35.3\% of the genitourinary system and $29.4 \%$ involved the cardiovascular system). $23.8 \%$ of cases were part of a syndrome, 13 and 18 trisomy were the most common (12 cases, $18.5 \%)$. 13 newborns died $(23.2 \%), 8$ of them $(61.5 \%)$ had IUGR. Conclusion: The presence of UUA in a foetus or newborn represents a high risk for IUGR, MCMF, chromosome anomalies, prematurity and increase of neonatal morbidity and mortality.

\section{KEY WORDS: Single umbilical artery, congenital malformations}

\section{INTRODUCCIÓN}

El cordón umbilical está formado por dos arterias, una vena, un alantoides rudimentario, el residuo del conducto onfalomesentérico y la gelatina de Warton (1). Durante el período embrionario los vasos se forman a partir del mesodermo que reviste el saco vitelino desde los 18 días. A los 23 días ya existe un sistema circulatorio con un sistema arterial formado por dos arterias aortas que al entrar al pedículo se denominan arterias umbilicales (2). Los mecanismos etiopatogénicos de la arteria umbilical única (AUU) no están claros, pero se ha propuesto que podría producirse por aplasia o atresia de una de las dos arterias o por persistencia de la AUU presente normalmente en las etapas tempranas del desarrollo embrionario que no evoluciona hacia la diferenciación de la segunda arteria (3).

La presencia de los tres vasos puede verse por ecografía a partir de la $12^{\mathrm{a}}$ semana de gestación. AUU es una de las malformaciones congénitas más frecuentes en el recién nacido con una incidencia variable alrededor de $1 \%$ de todos los nacimientos. La arteria umbilical izquierda falta con mayor frecuencia que la derecha $(4,5)$.

Entre un 30 y $60 \%$ de los RN con AUU presentan otras malformaciones estructurales asociadas $(4,6)$. También pueden presentarse anormalidades cromosómicas, tanto aneuploidías como trisomías 18 y 13 $(7,8,9,10)$. La trisomía 21 se asocia con mucho menor frecuencia con AUU (4). Tanto el examen prenatal ecográfico como el examen físico del RN deben incluir la visualización de los vasos del cordón.

Otro hecho comprobado es que AUU aumenta el riesgo para que el feto presente restricción del crecimiento intrauterino (RCIU) (6) al igual que un aumento de la morbimortalidad neonatal debido a la asociación con malformaciones fetales y aneuploidías.

\section{MATERIAL Y MÉTODO}

El Hospital Clínico de la Universidad de Chile
( $\mathrm{HCUCH})$ es miembro del Estudio Colaborativo Latino Americano de Malformaciones Congénitas (ECLAMC) desde 1969. Ingresan al registro todos los recién nacidos (RN) vivos (RNV) y mortinatos (RNM) de peso de nacimiento igual o superior a 500 gramos, que presenten una o más malformaciones. Se completa una ficha ad-hoc y se toma como control al RN del mismo sexo que nace a continuación y que no presente malformaciones.

Los objetivos de este trabajo son establecer la prevalencia al nacimiento de AUU y compararla con la obtenida anteriormente, registrar la cuantía de las asociaciones con otras malformaciones congénitas y aneuploidías, así como conocer la magnitud de la morbilidad y mortalidad neonatal de estos RN. Comprobar la relación del RCIU y de la prematuridad con esta anomalía.

\section{RESULTADOS}

En el período estudiado, mayo de 1998 a junio de 2010, ocurrieron en la maternidad del Hospital Clínico de la Universidad Chile 22.011 nacimientos consecutivos, 21.825 fueron nacidos vivos y 186 mortinatos, lo que representa una mortalidad fetal tardía o mortinatalidad de $0,84 \%$.

En esta muestra se encontraron 65 RN que sólo tenían dos vasos en el cordón umbilical, una arteria y una vena, lo que representa una prevalencia al nacimiento de AUU de $0,3 \%$. De ellos $56 \mathrm{RN}$ fueron nacidos vivos $(86,2 \%)$ y 9 mortinatos $(13,8 \%)$. No hubo diferencias de frecuencia por sexos, 30 fueron masculinos, 32 femeninos y 3 con ambigüedad sexual.

Al comparar algunos antecedentes demográficos de los casos y de sus controles (Tabla I), observamos que el promedio de edad materna fue 30,7 años y el promedio de edad paterna de 33,5 años. En los controles el promedio de edad materna fue 29,7 años y el de edad paterna 32,5 años, diferencia no significativa. 
Tabla I

COMPARACIÓN DE TRES VARIABLES DEMOGRÁFICAS EN RECIÉN NACIDOS CON AUU Y SUS CONTROLES

\begin{tabular}{lccr}
\hline Variables & AUU (n: 65) & Controles $(\mathrm{n}: 63)$ & Valor $p$ \\
\hline Peso al nacer $(\mathrm{g})$ & $\mathrm{X} \pm \mathrm{DE}$ & $\mathrm{X} \pm \mathrm{DE}$ & $<0,0001$ \\
Edad gestacional $(\mathrm{s})$ & $2.309 \pm 1.219,5$ & $3.364,3 \pm 505$ & $<0,0001$ \\
Edad materna $(\mathrm{a})$ & $35 \pm 4,3$ & $38,4 \pm 1,3$ & $\mathrm{NS}$ \\
\hline
\end{tabular}

X: promedio. DE: desvío estándar. AUU: arteria umbilical única. NS: no significativo.

El promedio de peso de nacimiento de los casos fue de 2.309 gramos, y en los controles de $3.364,3$ gramos $(p<0,0001)$. El promedio de edad gestacional en los casos fue 35 semanas y en controles de 38,4 semanas $(p<0,0001)$.

En los casos, $30(46,2 \%)$ fueron pequeños para la edad gestacional (PEG), $26(40 \%)$ adecuados para la edad gestacional (AEG) y $9(13,8 \%)$ grande para la edad gestacional (GEG); en tanto que en los controles hubo 5 (7,9\%) PEG, 52 (79,4\%) AEG y $8(12,7 \%)$ GEG, $(p<0,0001)$. $36 \mathrm{RN}(55,4 \%)$ eran prematuros.

En los casos, presentaron enfermedades agudas durante el embarazo 39 madres $(60 \%)$ y 27 enfermedades crónicas $(41,5 \%)$ (Tabla II). Entre las enfermedades agudas destacaron enfermedades respiratorias en 11 casos, infección del tracto urinario (ITU) en 7 casos y síndrome hipertensivo del embarazo en 5 casos (Tabla III). Entre los controles, $21(32,3 \%)$ presentaron enfermedades agudas ( $p=0,0013)$ (Tabla III) y $27(41,5 \%)$ enfermedades crónicas $(p=0,98)$ (Tabla IV).

Tabla II

ANTECEDENTES MATERNOS EN RECIÉN NACIDOS CON AUU Y SUS CONTROLES

\begin{tabular}{lcc}
\hline Antecedentes & AUU (n: 65) & Controles (n:63) \\
\hline $\begin{array}{l}\text { Pequeño para la edad } \\
\text { gestacional }\end{array}$ & 30 & $5^{\star}$ \\
Enfermedades agudas & 39 & 21 \\
Enfermedades & 27 & 27 \\
crónicas & & \\
Otros malformados en & 25 & 7 \\
la familia & 10 & 1 \\
Metrorragia & 9 & 13 \\
Abortos previos & & \\
\hline
\end{tabular}

AUU: arteria umbilical única. ${ }^{\star} p<0,0001$.
Tabla III

ENFERMEDADES AGUDAS PRESENTADA POR LAS MADRES CON RECIÉN NACIDOS CON AUU Y SUS CONTROLES DURANTE EL EMBARAZO

\begin{tabular}{lcc}
\hline Enfermedad & AUU & Controles \\
\hline Enfermedad respiratoria & 11 & $3^{*}$ \\
Infección del tracto urinario & 6 & 1 \\
Síndrome hipertensivo del & 5 & 7 \\
embarazo & & \\
Diabetes gestacional & 4 & 10 \\
Dolor (dental, neuralgia & 2 & 0 \\
trigémino) & & \\
Apendicitis aguda & 1 & 0 \\
Colecistectomía & 1 & 0 \\
Gastroenteritis & 1 & 0 \\
\hline
\end{tabular}

AUU: arteria umbilical única. * $p<0,05$

Tabla IV

ENFERMEDADES CRÓNICAS PRESENTADAS POR LAS MADRES CON RECIÉN NACIDOS CON AUU Y SUS CONTROLES DURANTE EL EMBARAZO

\begin{tabular}{lcc}
\hline Enfermedad & AUU & Controles * \\
\hline Tabaquismo crónico & 14 & 14 \\
Obesidad mórbida & 3 & 1 \\
Hipotirpoidismo & 3 & 2 \\
Asma bronquial & 2 & 1 \\
Hipertensión arterial crónica & 2 & 3 \\
Diabetes mellitus tipo 2 & 2 & 2 \\
Várices & 1 & 0 \\
Mioma uterino & 1 & 0 \\
\hline
\end{tabular}

* Diferencias no significativas. 
Presentaban antecedentes de tabaquismo crónico 14 casos $(21,5 \%)$ y $14(21,5 \%)$ en los controles $(p=0,8)$. El antecedente de metrorragia del primer trimestre estaba presente en 10 de los casos $(15,4 \%)$ y $1(1,6 \%)$ entre los controles $(p<0,00001)$. En 25 casos $(38,5 \%)$ existía el antecedente de otro malformado en la familia y 7 en los controles $(11,1 \%)(p=0,0003)$.

En $26(40 \%)$ pacientes la AUU se presentó en forma aislada, sin tener otra malformación asociada, $10(40 \%)$ de ellos presentaron RCIU y 1 falleció $(3,8 \%)$ que era un prematuro con RCIU severo.

En 39 pacientes $(60 \%)$ la AUU estaba asociada a otras malformaciones (Tabla V); 17 casos $(43,6 \%)$ pueden considerarse como malformaciones asociadas, no sindrómicas. De ellos, 15 eran nacidos vivos y 2 mortinatos. De los nacidos vivos fallecieron 3 (20\%). La letalidad de este grupo (mortinatos más mortineonatos) fue de $29,4 \%$. Entre las malformaciones asociadas más frecuentes encontradas están las del aparato genitourinario $(18,4 \%)$, las malformaciones de extremidades $(15,4 \%)$, las gastrointestinales $(12,3 \%)$, las cardiovasculares $(10,7 \%)$, y las del sistema nervioso central $(7,7 \%)$.

En 22 pacientes $(23,8 \%)$ la AUU era parte de un síndrome: 8 trisomías $13(12,7 \%) ; 4$ trisomías $18(6,3 \%) ; 4$ holoprosencefalia $(6,3 \%) ; 3$ secuencia de Potter (4,6\%) 1 síndrome de tallo corporal, y 1 caso de síndrome Cri du Chat. De estos $22 \mathrm{RN}$, 16 fueron NV $(72,7 \%)$ y 6 mortinatos $(27,3 \%)$. De los NV fallecieron $11(68,8 \%)$ y 15 de ellos $(68,2 \%)$ presentaban RCIU, por lo que sólo 5 fueron dados de alta vivos $(22,7 \%)$. La letalidad en este grupo (mortinatos más mortineonatos) fue $77,2 \%$.

Del total de 56 nacidos vivos, fallecieron 13 (23,2\%), 8 (61,5\%) de los cuales tenían RCIU; 42 $\mathrm{RN}$ fueron dados de alta vivos, lo que representa una sobrevida de $75 \%$. Hubo 1 caso $(1,6 \%)$ en que

\section{Tabla V \\ MALFORMACIONES PERINATALES ASOCIADAS A AUU}

\begin{tabular}{lcc}
\hline Malformaciones & $\mathrm{n}$ & $\%$ \\
\hline Genitourinarias & 12 & 18,4 \\
Extremidades & 10 & 15,4 \\
Gastrointestinales & 8 & 12,3 \\
Cardiovasculares & 7 & 10,7 \\
Sistema nervioso central & 5 & 7,7 \\
Pulmonares & 1 & 1,5 \\
\hline
\end{tabular}

AUU: arteria umbilical única. había consanguinidad parental. Cinco $(7,7 \%)$ quedaron embarazadas estando tomando anticonceptivos orales.

El promedio de ecografías prenatales fue de 5,14 . El diagnóstico prenatal de AUU se hizo en 40 pacientes $(61,5 \%)$. En 11 pacientes el estudio citogenético fue anormal.

\section{DISCUSIÓN}

En este trabajo se estudia la prevalencia al nacimiento de AUU y la de las malformaciones asociadas que la acompañan en los recién nacidos de la maternidad del Hospital Clínico de la Universidad de Chile, en el período mayo de 1998 a junio de 2010, como una continuación de otro trabajo nuestro que comprometió al período de enero de 1978 a abril de 1998 (7), con el objetivo de completar 32 años de vigilancia epidemiológica.

El significado clínico de la presencia de AUU no está aún claramente establecido. Según algunos autores la asociación con anomalías estructurales llega a 33\% (4). En nuestra muestra, la AUU se asoció con otras malformaciones en $60 \%$ de los casos. El porcentaje de aneuploidías alcanza el $10 \%$ $(8,9,10)$, mientras que en nuestro estudio el $19 \%$ de los casos eran aneuploidías.

La prevalencia de AUU encontrada en nuestra muestra $(0,3 \%)$ es muy superior a la encontrada por nosotros en una comunicación anterior (7) para el período $1978-98(0,1 \%)$, pero muy inferior a la comunicada por otros autores que dan entre $0,6 \%$ y $1,2 \%(11,12,13)$. Hua y cols $(14)$, en una casuística de 72.373 embarazos da una prevalencia de $0,61 \%$ y Horton y cols de 1,3\% (6). La explicación podría estar en que haya un subregistro nuestro, tanto en diagnóstico ecográfico prenatal; sólo el $61,5 \%$ de los casos fueron diagnosticados prenatalmente y también postnatal.

Los hallazgos de este estudio muestran que los niños que presentan AUU tienen un riesgo aumentado para RCIU lo que se manifiesta al nacimiento por ser PEG. El 46,2\% de los RN de nuestra muestra presentaron RCIU. La alta incidencia de esta alteración del desarrollo fetal en los niños con AUU es significativamente mayor que la encontrada anteriormente por nosotros (9), 41,8\% para el período 1978-98. Muestra además una alta asociación con malformaciones congénitas mayores (60\%). Esta concordancia demuestra, una vez más, la importancia que la RCIU tiene como factor de riesgo para malformaciones congénitas estructurales, aneuploidías y aberraciones cromosómicas. Gran importancia también tiene el hecho de el alto porcentaje de prematuros en nuestra muestra $(55,4 \%)$, aún 
cuando otros autores no encuentran diferencias en la prematuridad entre los niños con AUU y los normales (15). Nuestros hallazgos, sin embargo, están plenamente de acuerdo con lo reportado por otros autores (7). Estos cuatro importantes hechos: asociación con anomalías estructurales mayores, anormalidades cromosómicas y aneuploidías, RCIU y prematuridad, deben obligar a realizar ecografías seriadas y extremar las medidas de control del embarazo cuando se hace el diagnóstico prenatal de AUU, por el alto riesgo de asociación con esas patologías (16).

\section{BIBLIOGRAFÍA}

1. Moore KL. Aparato circulatorio. En: Moore $\mathrm{KI}(\mathrm{Ed})$. Embriología Clínica. México. Nueva Editorial Interamericana. 1985. pp 78-80.

2. Illanes J, Montenegro MA. Sistema cardiovascular. En: Montenegro MA, Mena MA, Illanes J, Lemus D. Embriología Clínica. Chile. Editorial Universitaria. 1996. pp 231.

3. Monie IW. Genesis of single umbilical artery. Am J Obstet Gynecol 1970;108:400-5.

4. Robinson JN, Muhamad AZ. Anomalías de la pared abdominal y del cordón umbilical. Clin Perinatol 2000;4:987-8.

5. Abuhamad AZ, Shaffer W, Mari G, Copel JA, Hobbins JC, Evans AT. Single umbilical artery: Does it matter which artery is missing? Am J Obstet Gynecol 1995;173:728-32.

6. Horton AL, Barroilhet L, Wolfe HM. Perinatal outcomes in isolated single umbilical artery. Am J Perinatol 2010;27:321-4.

7. Austin-Warf ED, Nazer J, Castillo S, Llanos C. Arteria umbilical única y malformaciones congénitas asociadas: Experiencia en el hospital Clínico de la Universidad de Chile. Rev Chil Pediatr 1998;69:195-9.

8. Granese R, Coco C, Jeanty P. The value of single umbilical artery in the prediction of fetal aneuploidy: findings in 12672 pregnant women. Ultrasound Q 2007; 23: 117-21.

9. Byrne J, Blanc WA. Malformations and chromosome anomalies in espontaneusly aborted fetuses with single umbilical artery. Am J Obstet Ginecol 1985;151:340-2.

10. Volpe G, Volpe P, Boscia FM, Volpe N, Buonadonna AL, Gentile L. "Isolated" single umblical artery: incidence, cytogenetic abnormalities, malformation and perinatal outcome. Minerva Ginecol 2005;57:189-198.

11. Gornall AS, Kurincsuk JJ, Konje JC. Antenatal detection of a single umbilical artery : Does it matter? Prenat Diagn 2003;23:117-23.

12. Mu SC, Lin CH, Chen YL, Sung TC, Bai CH, Jow GM. The perinatal outcomes of asymptomatic isolated single umbilical artery in full-term neonates. Pediatr Neonatol 2008;49:230-3.

13. Csécsei K, Kovács T, Hinchliffe SA, Papp Z. Incidence and associations of single umbilical artery in prenatally diagnosis malformed, midtrimester fetuses: a review of 62 cases. Am J Med Genet 1992;43:524-30.

14. Hua M, Odibo A, Macones G, Roehl K, Kimberly A, Crane J, Cahill A. Single umbilical artery and its associated findings. Obstet Ginecol 2010;115: 930-4.

15. Bombrys AE, Neiger R, Hawkins S. Pregnancy outcome in isolated single umbilical artery. Am J Perinatol 2008;25:239-42.

16. Wiegand S, McKenna DS, Croom C, Ventolini G, Jonek JD, Neiger R. Serial sonographic growth assessment in pregnancies complicated by an isolated single umbilical artery. Am J Perinatol 2008;25:149-52. 